\title{
Pupil-linked arousal is sensitive to subconscious processing of auditory novelty
}

\author{
Sergio Osorio ${ }^{1,3, *}$, Martín Irani ${ }^{2,3}$, Javiera Herrada $^{4}$ and Francisco Aboitiz ${ }^{1,3, *}$ \\ ${ }^{1}$ Laboratory for Cognitive and Evolutionary Neuroscience, Department of Psychiatry, Faculty of Medicine, Pontificia \\ Universidad Católica de Chile, Santiago de Chile. \\ ${ }^{2}$ Laboratory for Neurodynamics of Cognition, Department of Psychiatry, Faculty of Medicine, Pontificia Universidad \\ Católica de Chile, Santiago de Chile. \\ ${ }^{3}$ Interdisciplinary Center for Neuroscience, Pontificia Universidad Católica de Chile, Santiago de Chile \\ ${ }^{4}$ Biomedical Neuroscience Institute (BNI), Faculty of Medicine, Universidad de Chile, Santiago De Chile \\ *Corresponding authors: Sergio Osorio - srosorio@uc.cl, Francisco Aboitiz - faboitiz@uc.cl
}

The ability to detect novelty in sensory stimuli is at the base of autonomic and goal-directed behavior. Pupil size, a proxy of the Locus Coeruleus-Norepinephrine system, is sensitive to auditory novelty. However, whether this response reliably reflects conscious processing of novelty remains contentious. Here, we characterized pupil and electrophysiological responses during conscious and subconscious processing of auditory novelty by presenting participants deviant stimuli that were below and above their discriminatory thresholds. We found higher pupil responses to subthreshold targets that were not consciously perceived as deviant stimuli. Larger pupil size and dilation rates were associated to more negative Event-Related Potential values extracted from temporal, prefrontal and anterior cingulate regions. We suggest that increased phasic responses to deviant targets that escape conscious perception reflect Norepinephrine-mediated adaptation of arousal levels in order to meet the perceptual and behavioral demands imposed by the task at hand.

\section{Introduction}

The ability to extract regularities and detect novelty in the form of violations to the statistical properties of sensory information is of paramount importance for biological organisms, as it mediates both autonomic responses and goal-directed behavior (Ranganath and Rainer, 2003; Tiitinen et al., 1994; Sohoglu and Chait, 2016). Conscious processing of novel stimuli, contrast-based saliency and arousal levels can speed up, delay, or even suppress neuronal and behavioral responses (Töllner et al., 2011, Aston-Jones and Cohen, 2005, Vasey et al., 2018). Remarkably, neural populations have 
the ability of fine-tuning its properties and accommodating neuronal gain-modulation thresholds in order to meet environmental or task demands (Ferguson and Cardin, 2020). Such gain-modulation adaptation is mediated by the activity of the Locus Coeruleus - Norepinephrine (LC-NE) system in response to the demands imposed by environmental and task-specific conditions (Aston-Jones and Cohen, 2005; Poe et al., 2020). Additionally, pupil size has been shown to reflect NE-mediated arousal (Aston-Jones and Cohen, 2005; Ferguson and Cardin, 2020) and, more recently, to be sensitive to the detection of auditory novelty (Quirins et al, 2018; Zhao et al., 2019). However, whether this response requires conscious processing of sensory novelty remains contentious.

A phasic increase in pupil size has been associated with subjects' conscious processing of single auditory stimuli presentation, but not to auditory stimuli that are not consciously perceived and reported (Bala et al., 2019). Similarly, consciously reported violations of auditory regularities in tonal sequences elicit a pupil response during active-counting and passive listening, whereas violations that escape conscious perception do not elicit a pupil response (Quirins et al, 2018). This line of evidence suggests that the pupil reflects conscious processing of novel stimuli. In contrast, introducing two oddballs, each one of different saliency, suppresses the pupil response to the less salient target during passive engagement. Interestingly, requiring participants to report any detected novelty restores the pupil response to both targets (Liao et al., 2016A). Likewise, abrupt violations of auditory regularities but not sudden regularity emergence elicit an increase in pupil size during passive listening. However, asking participants to monitor any change in the auditory scene results in a pupil response to both regularity violation and regularity emergence (Zhao et al., 2019). This latter line of evidence therefore suggests that the phasic pupil response can operate independently of conscious perception and that behavioral relevance of perceived stimuli might be important in eliciting a pupil response.

Two well attested markers of conscious and subconscious processing of auditory novelty are the Mismatch Negativity (MMN) and the P3 positivity complex. Auditory stimuli that violate the predictions of the central auditory system elicit an MMN response peaking around 200 milliseconds after odd stimulus presentation. This Event-Related Potential (ERP) occurs independently of attentional state or conscious processing (Bekinschtein et al., 2009; Näätänen et al., 2007, 2019). Generators of this response have been identified in posterior superior and middle temporal and prefrontal regions (Garrido et al., 2009) and more recently, in anterior portions of the Cingulate Cortex, a region involved in error detection and the processing of surprisal (Hyman et al., 2017). The MMN response is proposed to reflect an orienting attention mechanism involved in bottom-up processing of sensory information (Näätänen et al., 2007, 2019). In turn, novel auditory events that are attended to and consciously detected elicit a positive deflection in the ERP, known as the P3 response, starting at around 300 milliseconds after the presentation of a novel stimulus (Polich, 2007; 
Kamp and Donchin, 2015). Multiple generators for this event have been reported within a frontocentro-parietal network encompassing dorsomedial prefrontal regions, precentral and postcentral gyri, superior parietal and cingulate regions (Linden, 2005). The P3 response has been proposed to reflect context-updating and memory-dependent information processing mechanisms (Polich, 2007).

In this study, we investigate how the pupil responds to auditory novelty with and without conscious perception, and how such response relates to well established markers of subconscious and conscious auditory processing, namely the MMN and the P3 ERP events. For this, we implemented a novel task which allowed disentangling conscious from subconscious processing of auditory novelty by presenting deviant targets above and below each subject's threshold for conscious discrimination. We found increased pupil responses to subthreshold deviant targets that were not consciously perceived in contrast to consciously processed suprathreshold targets. Increased pupil dilation responses were associated to more negative mean ERP values extracted from sourcereconstructed temporal, prefrontal and anterior cingulate regions during the latency time period corresponding to the MMN. We suggest that an increased pupil response to deviant targets that are not consciously perceived reflects an increased demand of NE which might be necessary in order to accommodate current arousal levels to the perceptual and behavioral demands imposed by the task at hand.

\section{Results}

\section{Subthreshold deviant targets are associated with high error rates and slower reaction times}

All participants performed a staircase procedure which allowed identifying their individual discriminatory thresholds before each block of the thresholded deviant detection task. The staircase procedure allowed setting subthreshold deviant targets adaptively and objectively according to the individual hearing abilities of each participant (Figure 1A). Participants $(n=24$, mean age $=25.5$, range $=13$ ) were binaurally presented sequences of narrowband sinusoidal tones and asked to decide whether the last tonal stimulus (i.e the target tone) was the same as or different from the previous standard tones (Figure 1B). The target stimulus could be either another standard tone (tgtSTD), a suprathreshold deviant (supraDEV) or a subthreshold deviant (subDEV). Because subDEV stimuli were deliberately intended to be below the threshold for conscious discrimination, subthreshold deviants were expected to be systematically judged as standard tones and should therefore be associated with high error rates. Conversely, target standards and suprathreshold deviants should be correctly and systematically identified as such, which should manifest as high accuracy rates. Asserting that participants conformed to this expected response pattern was important in order to guarantee that subthreshold deviants were subconsciously processed but not consciously perceived. 
Therefore, we tested individual accuracy rates for each block and for each set of stimuli within each condition against the chance probability using a binomial distribution test (Table S2, supplementary materials). Data from blocks that failed to meet the above-chance performance criterion (i.e. tgtSTD/correct, subDEV/incorrect, supraDEV/correct) were excluded from subsequent analyses. We confirmed that both target standards and subthreshold deviant targets were associated with high hit rates, whereas subthreshold deviant targets where associated with high error rates. (Figure 1C). Next,

112 we computed median Reaction Times (RTs) and performed group-level statistics. Due to the 1000-

113 millisecond delay in behavioral response, we did not expect to see a significant difference in median

114 reaction times across conditions. Interestingly, we found that the median reaction time to 115 suprathreshold deviant targets was statistically faster than to subthreshold deviant targets at the group116 level $(n=21, p<0.05$, Bonferroni-corrected, Figure 1D). No other statistical differences were found 117 between median RT.

A.

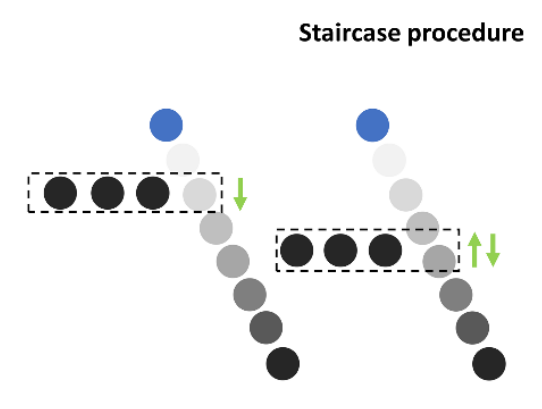

C.

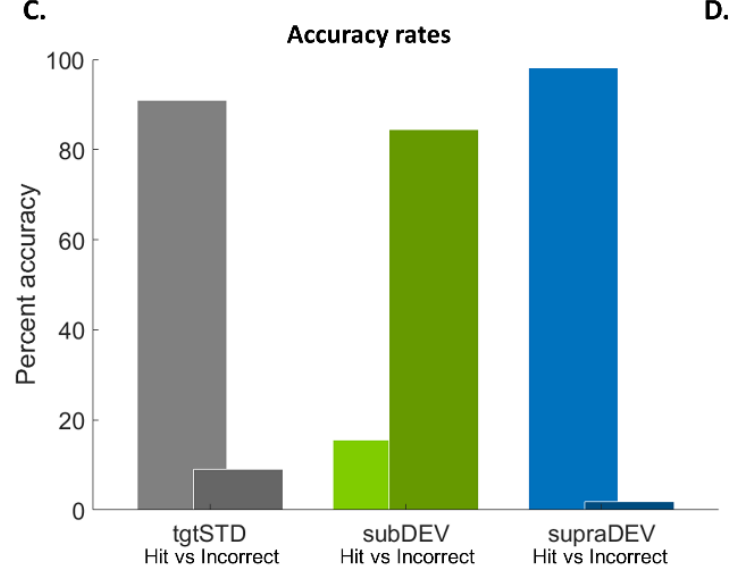

B.

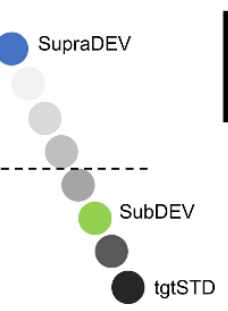

D.
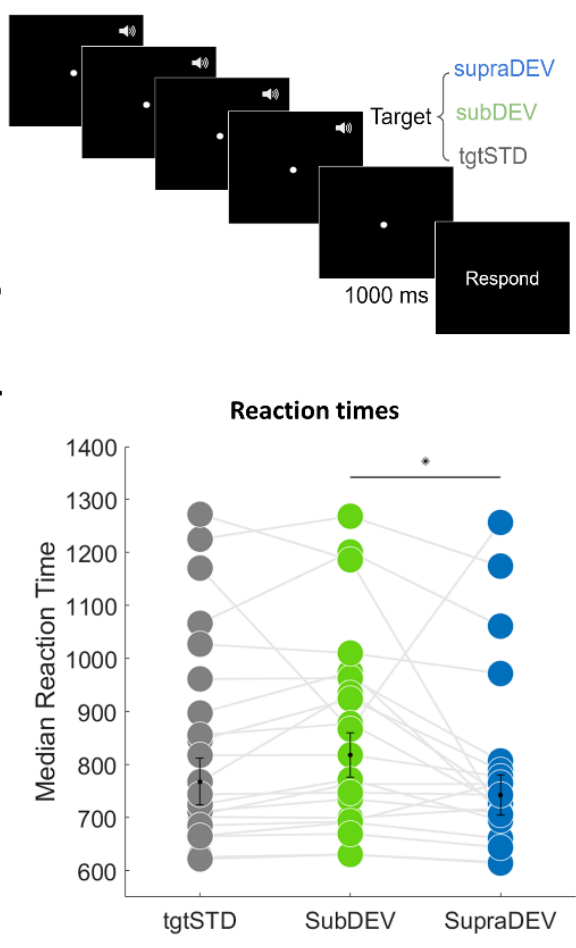

Figure 1. A) Schematic of the staircase procedure. Circles represented pure-narrowband tones. To detect the participant's discriminatory threshold, a train of standard tones was presented against a target tone. Participants were asked whether the last tone was the same or different from the previous ones. If the participant responded "different" the target tone was stepped down in 5hz. If the participants responded "same", the target tone was stepped-up in 5Hz (see methods). The threshold was defined as the point at which the participant could no longer discriminate between the standard and the target 
train of STD tones were followed by another STD, a SupraDEV or a SubDEV. Participants were instructed to respond whether the last tone was the same or different from the preceding ones by pressing one of two buttons when a prompt appeared on screen. C). Accuracy rates. Hits (light-shaded bars) and incorrect responses (dark-shaded bars) for the standard (gray), subthreshold (green) and suprathreshold (blue) target stimuli. All data were above the chance probability. D) Median reaction times for tgtSTD/correct, subDEV/incorrect and supraDEV/correct responses. Asterisk represents a statistically significant effect.

\section{Increased pupil response to subthreshold deviant targets that escape conscious perception}

Next, we investigated pupil responses to standard, subthreshold deviant and suprathreshold deviant targets. We computed two measures of phasic pupil change: normalized pupil size and normalized pupil rate of change. We decided to include pupil rate of change as it provides more timeresolved information about both pupil dilation and constriction compared to pupil size. As a control procedure, we inspected the nature of the relationship between pupil size and pupil rate of change during our 1000-millisecond time window of interest. We confirmed that an increased pupil size was associated with faster dilation of the pupil whereas a smaller pupil size was associated either with slower dilation or constriction of the pupil across conditions (Figure S3, supplementary materials).

If the pupil reliably reflected conscious processing of auditory novelty, we would expect to see an increased pupil response to suprathreshold targets that were consciously perceived, but not to subthreshold targets which escaped conscious perception. In contrast with this expectation, we found that subthreshold deviants were associated with increased pupil sizes compared to standard and suprathreshold targets (Figure 2A). This effect was significant between $\sim 180$ and $\sim 540$ milliseconds after target stimulus onset compared to suprathreshold deviant targets $(n=21, p<0.05$, Bonferronicorrected). Dilation rates were also statistically faster for subthreshold deviants between $\sim 100$ and $\sim 280$ milliseconds compared to target standards ( $\mathrm{n}=21, \mathrm{p}<0.05$, Bonferroni-corrected $)$ and between $\sim 130$ and $\sim 290$ milliseconds compared to suprathreshold targets $(\mathrm{n}=21, \mathrm{p}<0.05$, Bonferronicorrected, Figure 2B). No statistical difference was observed between pupil responses to standards and suprathreshold deviants. These results therefore suggest that the pupil response does not reliably reflect conscious processing of novel auditory stimulus.

We also investigated whether pupil responses were related to reaction times. We found that subjects who showed bigger pupil sizes also showed slower reaction times between 0 and 900 milliseconds across conditions (tgtSTD: $\mathrm{n}=17$, rho $=0.629, \mathrm{p}=0.008$; subDEV: $\mathrm{n} 17,0.544, \mathrm{p}=$ 0.026 ; supraDEV $=\mathrm{n}=17$, rho $=0.580, \mathrm{p}=0.016$, Figure $2 \mathrm{C}$ ). As for pupil rate of change, we observed that faster dilation rates were also associated with slower reaction times for standard $(\mathrm{n}=17$, rho $=0.676, \mathrm{p}=0.003)$ and suprathreshold deviant targets $(\mathrm{n}=17$, rho $=0.561, \mathrm{p}=0.021$, Figure 2D), but not for subthreshold targets. 
A.

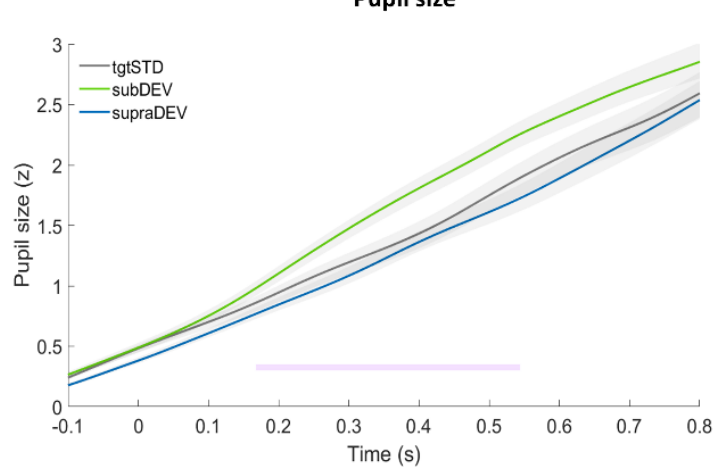

c.

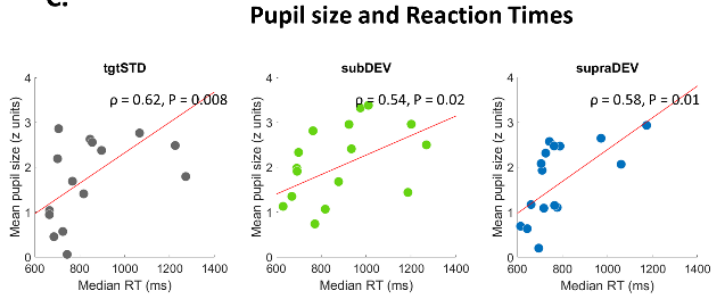

B.

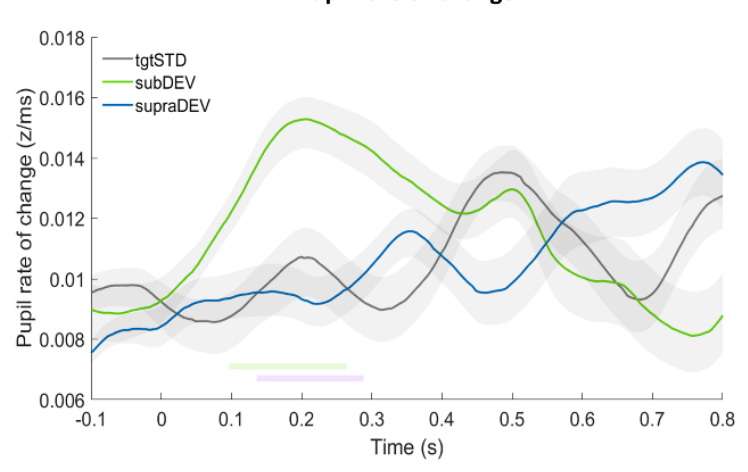

D.

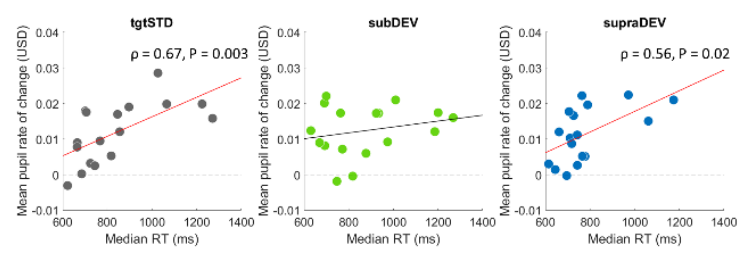

Figure 2. A) Mean pupil size in response to target standards (gray line), subthreshold deviant targets (green line) and suprathreshold deviant targets (blue line). Gray shaded areas represent the 95\% Confidence Interval (C.I.) for the Standard Error of the Mean (S.E.M.) over time calculated using a studentized bootstrapping procedure. The purple line indicates a significant difference between subDEV and supraDEV targets. B) Mean pupil rate of change calculated as the derivative of pupil size (in z units per second). Gray shaded areas represent the 95\% C.I. for the S.E.M. Green and purple lines indicate a significant effect between subtDEV and tgtSTD and between subDEV and supraDEV targets correspondingly $(\mathrm{n}=21, \mathrm{p}$ $<0.05$, Bonferroni-corrected). C) Non-parametric spearman Correlation between pupil size and median reaction time for tgtSTD (left) subDEV (middle) and supraDEV (right). Red least-square lines represent a statistically significant effect. D) Non-parametric spearman Correlation between pupil rate of change and median reaction time for tgtSTD, subDEV and supraDEV.

\section{ERP responses reflect subconscious processing of subthreshold targets and conscious processing} suprathreshold targets

We then investigated the mean ERP response measured at the scalp level to the three types of target stimuli. If the MMN is independent of conscious perception, we would expect to observe an MMN to both subthreshold deviant targets incorrectly judged as standard tones and suprathreshold deviant targets correctly reported as such. Additionally, if the P3 reflects conscious processing of sensory novelty, we should expect to see a P3 response to consciously detected suprathreshold deviant targets, but not to subthreshold deviants. In line with these expectations, both subthreshold and suprathreshold deviants elicited an MMN neural response at electrode $\mathrm{Cz}$ (Figure 2A). This effect was significant between $\sim 180$ and $\sim 195$ milliseconds for subthreshold deviant targets and between $\sim 130$ and $\sim 196$ milliseconds for suprathreshold deviant targets $(\mathrm{n}=23, \mathrm{p}<0.05$, Bonferroni 
corrected). In contrast, we observed a P3 response only to suprathreshold deviant targets that were consciously perceived (Figure 2A). This effect was significant between a wider time window comprising $\sim 220$ and $\sim 440$ milliseconds after stimulus presentation $(\mathrm{n}=23, \mathrm{p}<0.05$, Bonferroni corrected). Our ERP results thus suggest that subthreshold deviants were subconsciously processed but escaped conscious perception, whereas suprathreshold deviant targets were both subconsciously and consciously processed.

\section{EEG-source imaging reveals involvement of temporal, prefrontal and anterior cingulate regions}

In order to inspect the cortical activation dynamics associated to conscious and subconscious processing of auditory novelty, we projected each participant EEG signals onto template cortical surfaces. We performed group analyses for the difference in means between both types of deviant targets (subDEV and supraDEV) against target standards. Unconstrained forward models using the Minimum Norm solution and source imaging using the sLORETA method on MNI/ICBM152 surface templates showed patterns of activation consistent with previously reported cortical origins of the MMN and the P3 ERP responses (Figures 3B and 3C).

For subthreshold deviant targets, we observed increased activation of left prefrontal (DLPFC), left pre and postcentral and right temporo-parietal regions at 192 milliseconds. This prefrontal activation is consistent with reports of a frontal generator for the MNN response. At 300 milliseconds, subthreshold deviants elicited increased activation of right superior and middle temporal (STG and MTG) regions, as well as the right insula, but no activation was found for central, superior parietal or dorsomedial (DMPFC) regions which are classically associated with the P3 event (Figure 3B). These cortical activation dynamics are in line with the known origins of the MMN in temporal and prefrontal regions. For suprathreshold deviant targets, we found increased activation of insular, superior and middle temporal (STG and MTG), and prefrontal regions (DLPFC) bilaterally at 192 milliseconds. At 300 milliseconds, there was increased activation of dorsomedial prefrontal regions (DMPFC), superior pre and postcentral and superior parietal areas for suprathreshold deviant targets (Figure 3C). These latter results are consistent with the known cortical generators of the P3.

Finally, we found increased activation of the ACC for both subthreshold and suprathreshold deviants against target standards. For subthreshold deviants, there was an involvement of the ACC at between $\sim 220$ and $\sim 228$ milliseconds (Figure 3D). Suprathreshold deviants also elicited activation of the ACC between 220 and 232 milliseconds, but there was also involvement of the ACC between $\sim 328$ and $\sim 360$ milliseconds (Figure 3E). These results confirm the involvement of temporal, prefrontal and cingulate regions during the latency period corresponding to the MMN and of dorsomedial and central regions during the time window corresponding to the P3. 
A.

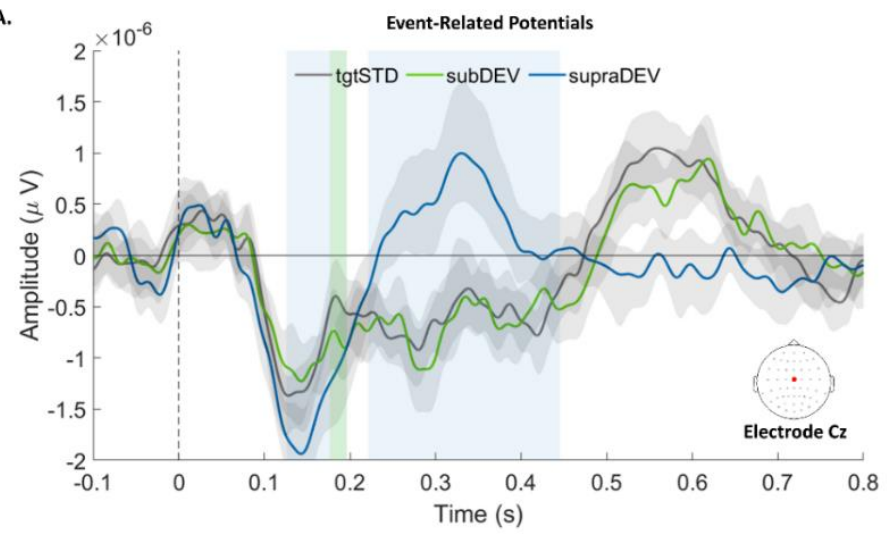

B.

Bifference in Means (SubDEV - tgtSTD)
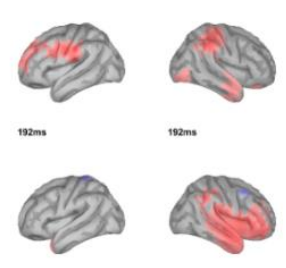

sooms

D.
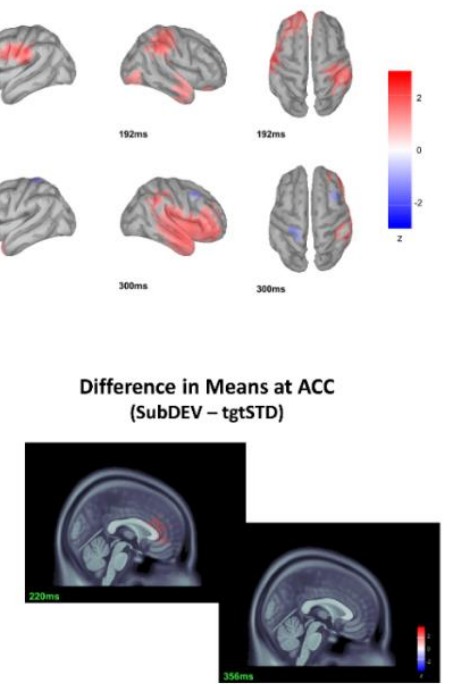

c.

$$
\text { Difference in Means }
$$
(SupraDEV - tgtSTD)
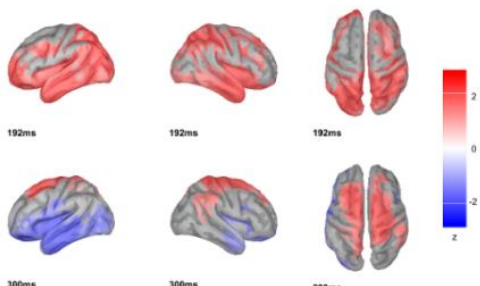

E.

Difference in Means at ACC (SupraDEV - tgtSTD)

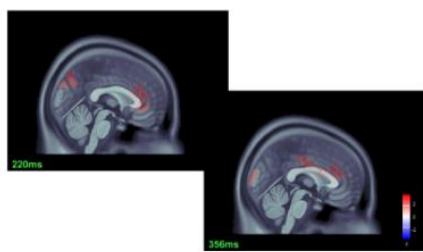

Figure 3. A) Mean ERP response at electrode $\mathrm{Cz}$ for target standards (gray line), subthreshold deviants (green line) and suprathreshold deviants (blue line). Light gray shaded area represents the studentized bootstrapped $95 \%$ C.I. of the mean. Blue shaded area represents time windows of statistical significance between suprathreshold deviant targets against standard tones. Green shaded area represents statistical significance for subthreshold deviant targets compared to standards. B) and C) Difference of means in projected EEG signals onto ICBM-152 template cortical surfaces (see Suppelemtary materials for full z-map movie clips). D) and E) Projection of the EEG signal into template fMRI volumes for subthreshold (D) and suprathreshold deviant targets at 220 and 356 milliseconds.

\section{Increased pupil responses are associated to more negative source-reconstructed ERP values}

Finally, we investigated the relationship between the pupil response and ERPs extracted from six source-reconstructed regions of interest (ROIs). These regions were informed by the literature on the generators of the MMN and P3 and our source-imaging results, and included left and right STG, left and right DLPFC, DMPFC bilaterally and ACC. We performed correlational analyses between 
234 our measures of pupil change and the mean ERPs extracted from our ROIs for two time windows of 235 interest corresponding to our ERP events of interest, namely the MMN response (150-220 ms) and 236 the P3 (280-320 ms) response. If the pupil response and the P3 were related neural events reflecting 237 context updating and memory-dependent information processing, we should see that subjects 238 showing more positive ERP values extracted from bilateral DMPFC or ACC within 280 and 320 239 milliseconds would also show increased pupil responses. Alternatively, if the pupil response and the 240 MMN were related neural events reflecting bottom-up orienting attention mechanisms, we would 241 expect to see that subjects showing more negative ERP values extracted from the STG, the DLPFC 242 and the ACC within 150 and 220 milliseconds would also show increased pupil responses.

243 We found evidence for the second scenario: for subthreshold targets, faster pupil dilation 244 rates were associated with more negative ERP values at right STG $(\mathrm{n}=17$, rho $=-0.497, \mathrm{p}=0.044$, 245 Figure 4A) and right DLPFC $(n=17$, rho $=-0.502, \mathrm{p}=0.041$, Figure 4B) during the 150-to-220246 millisecond time window, but this effect was absent between 280 and 320 milliseconds (Figure S4A 247 and S4B, supplementary materials). Also for subthreshold targets, a faster rate of change ( $\mathrm{n}=17$, rho $248=-0.664, p=0.004$, Figure $4 C)$ and bigger pupil size $(n=17$, rho $=-0.750 . p=0.008$, Figure 4D) 249 were associated to more negative ERP values extracted from the ACC between 280 and 320 250 milliseconds. This effect was not observed during the 150-220-millisecond time window (Figure S4C, 251 supplementary materials). Moreover, no effects were found for standard or suprathreshold deviant 252 targets. These findings suggest that subconscious processing of auditory novelty is associated with 253 both increased pupil response and more negative values in ERPs extracted from regions and time 254 periods corresponding to the $\mathrm{MMN}$ and the $\mathrm{P} 3$. 

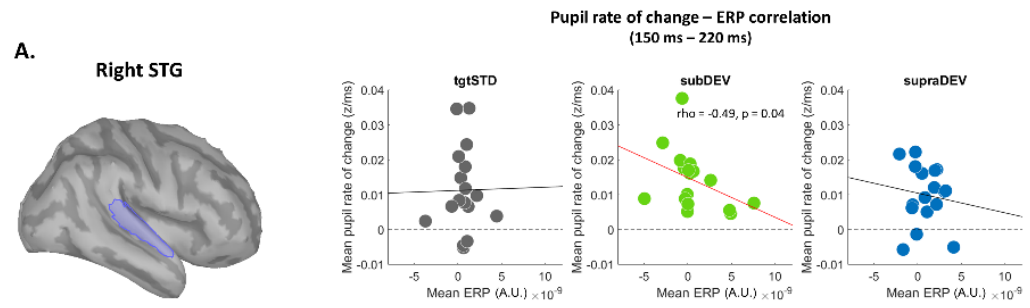

B.
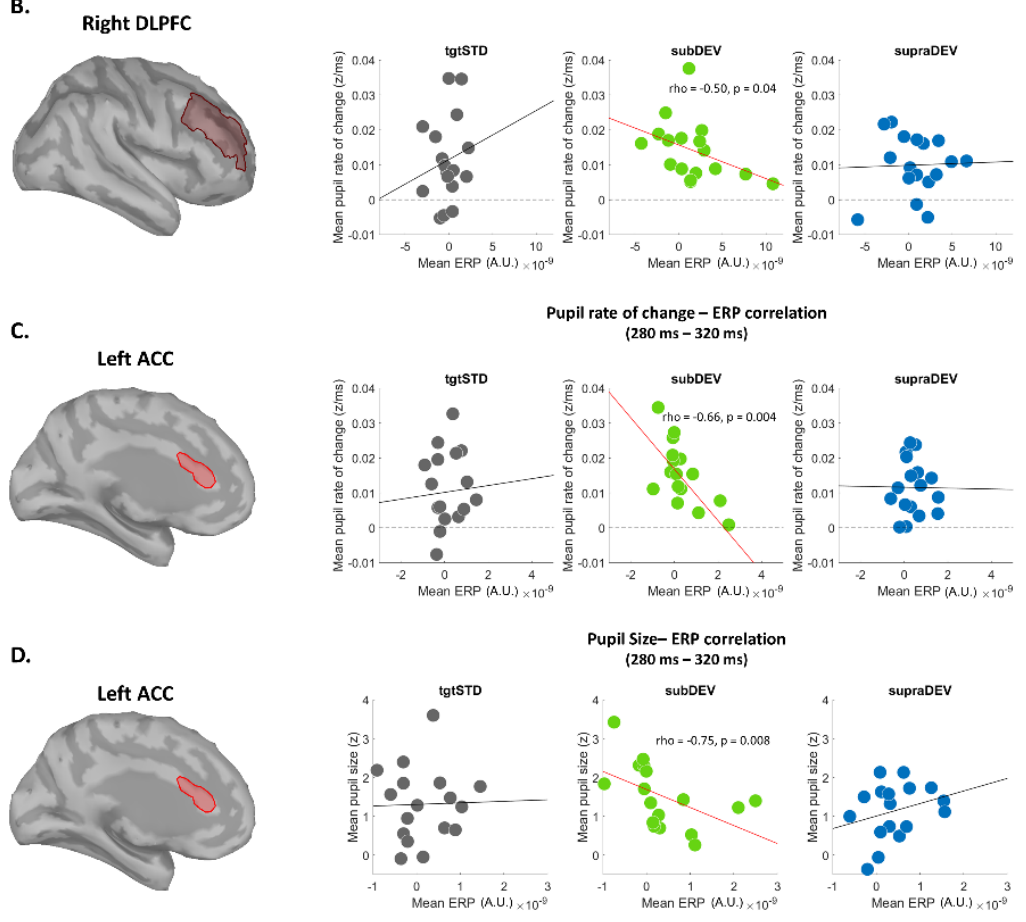

Figure 4. Non-Parametric spearman correlations between measures of pupil change and source-reconstructed ERP signals. Red least-square lines represent statistically significant effects. A) Correlation between mean ERP values extracted from the right Superior Temporal Gyrus (sSTG) and mean pupil rate of change between 150 and 220 milliseconds. B) Correlation between mean ERP values extracted from the right Dorsolateral Prefrontal Cortex (DLPFC) and mean pupil rate of change between 150 and 220 milliseconds after stimulus presentation. C) Correlation between mean ERP values extracted from the left Anterior Cingulate Cortex (ACC) and mean pupil rate of change between 280 and 320 milliseconds D) Correlation between mean ERP values extracted from the left Anterior Cingulate Cortex (ACC) and mean pupil size between 280 and 320 milliseconds.

\section{Discussion}

In this study, we investigated the pupil response during the processing of auditory novelty with and without conscious perception and how it relates to well established markers of subconscious and conscious auditory processing. Phasic changes in pupil size have been associated to a myriad of cognitive functions, including effort, saliency, arousal, attention, memory, consciousness among many others (van der Wel and van Steenbergen, 2018; Wang et al., 2014; 2018; Wainstein et al., 
2017; Clewett et al, 2020). This, however, has made it difficult to arrive to an overarching account of what the pupil reflects across cognitive domains.

Some studies have provided evidence that the pupil reflects conscious processing of detected sensory deviance during the processing of auditory novelty (Bala et al., 2019; Quirins et al., 2018). However, other results suggest that the pupil response can operate independently of conscious processing under certain task conditions, thus highlighting the role of behavioral relevance of perceived stimuli (Liao et al., 2016A; Zhao et al., 2019; Alamia et al., 2019). Our results add up to the latter line of evidence. Increased pupil size and dilation rates were observed in response to subthreshold deviant tones that escaped conscious perception and for which there was not an associated P3 response. In contrast, no pupil response was observed for suprathreshold deviants that were consciously identified and that elicited a P3 response. This suggests that the pupil does not reliably reflect conscious processing of detected deviance, as certain task conditions can elicit a pupil response in the absence of conscious perception.

Other studies have previously found that the pupil is sensitive to contrast-based stimulus saliency, and that a more pronounced pupil response is associated to increased contrast between standard and deviant stimuli (Liao et al., 2016B; Wang et al., 2014). Similar modulatory effects of contrast-based saliency have been found for the MMN (Näätänen et al., 2007) and the P3 (Teixeira et al., 2010; Texeira et al., 2014). If contrast-based saliency was driving pupil responses, we should have seen a negative correlation between pupil response and MMN across conditions, and a positive correlation between pupil response and P3 for suprathreshold deviants. However, we observed a pupil response to the least salient of both deviant targets, whereas no such response was observed to the most salient one. Moreover, we did not observe linear relationships between measure of pupil change and the MMN across conditions, or between pupil and P3 for suprathreshold deviants. This suggests that under our experimental conditions, pupil response was not modulated by contrast-based saliency.

Both subthreshold deviants and suprathreshold deviants elicited an MMN response at $~ 200$ milliseconds after target presentation. In contrast, suprathreshold deviants but not subthreshold deviants elicited a P3 response. This confirms that the staircase procedure along with our thresholdeddeviant detection task effectively resulted in subconscious and conscious processing of auditory novelty, in line with other adaptations to the classical oddball task, such as the global-local paradigm (Bekinschtein et al., 2009). Moreover, our results replicate findings which demonstrate that the MMN operates independently of attentional states and conscious perception, whereas the $\mathrm{P} 3$ necessitates the subject's conscious access to the target stimulus (Bekinschtein et al., 2009; Näätänen et al., 2019; Polich, 2007; Kamp and Donchin, 2015). EEG source-imaging confirmed that these signals originated from regions classically associated to the $\mathrm{MMN}$ and the $\mathrm{P} 3$, including middle temporal, superior 
temporal, prefrontal, dorsomedial and centro-parietal regions (Garrido et al., 2009; Linden, 2005). This reassures us that in spite of using a novel task, the events we observed indeed correspond to the classical ERP events associated to conscious and subconscious processing of auditory novelty. We also found evidence for the involvement of Cingulate and Insular regions, which is in line with more recent studies on surprisal and mismatch/error detection (Hyman et al., 2017; Citherlet et al., 2019; Han et al., 2019). Such results highlight the importance of these cortical areas during the processing of sensory novelty.

Previous studies have failed to identify a straightforward relationship between the pupil response and ERPs measured at the scalp level, particularly the P3 (Steiner and Barry, 2011; Kamp and Donchin, 2015). This is surprising because both the phasic pupil and the P3 responses are modulated by the activity of the LC-NE system (Aston-Jones and Cohen, 2005; Murphy et al., 2011, Vazey et al., 2018; Nieuwenhuis et al., 2005). Since we did not find evidence of such relationship, we also conclude that the pupil response and the P3 reflect separate neural mechanisms, even if they rely on a common neuromodulatory system. In contrast, we found evidence for a highly specific relationship between phasic pupil response and the MMN during subconscious processing of auditory novelty. A higher rate of change was associated to more negative ERP values computed from sourcereconstructed signals in right STG, right DLPFC and ACC between 150-220 milliseconds. These regions and time window overlap with the known generators and latency period of the MMN. Similarly, increased pupil size and faster dilation rates were associated to more negative ERP values extracted from the ACC between 280 and 320 milliseconds, a time window associated to the P3 event. Because the MMN has been associated to Glutamatergic and not to Noradrenergic modulation (Harms et al., 2020) and because the MMN reliably reflects whereas the pupil is sensitive to, but does not reliably reflect contrast-based saliency, we suggest that the pupil response and the MMN are both involved in orienting attention processes but still reflect different neural mechanisms. However, this effect was only observed for subthreshold deviant targets that escaped conscious perception and not for consciously processed suprathreshold targets.

What then does the pupil response reflect? A growing number of studies have associated phasic changes in pupil size to the adaptation of arousal levels by the activity of the LC-NE system (Urai et al., 2017; de Gee et al., 2014; Clewett et al., 2020; Krishnamurthy et al., 2017). Our observation that increased pupil size was associated to slower reaction times across conditions is reminiscent of the far-right tail of the Yerkes-Dodson curve (Aston-Jones and Cohen, 2005) and suggests that the pupil response was driven by changes in arousal levels ensuing the presentation of target stimuli. Moreover, previous studies have demonstrated that the phasic pupil response is associated to changes in global arousal levels which are driven by task-specific conditions and 
decision-making processes (Urai et al., 2017; de Gee et al., 2014). We consider that our findings are most interpretable in terms of changes in global arousal as a result of phasic LC-NE activity.

The Adaptive Gain Theory proposes that the function of phasic LC-NE system activation is to facilitate changes in arousal for the optimization of behavioral performance according to specific task demands (Aston-Jones and Cohen, 2005; Poe et al., 2020). Importantly, this theory discriminates between tonic LC-NE activation, which is associated with baseline LC firing and baseline arousal, and phasic LC-NE activation, which is associated to evoked LC firing and phasic arousal in response to stimulus-driven and task-relevant decision processes (Aston-Jones and Cohen, 2005; Gilzenrat et al, 2010; Poe et al., 2020). Phasic activation of the LC would result in the adaptation of neural gainmodulation functions thanks to increased NE input, which would in turn modulate cortical excitationinhibition balances, thus facilitating the adaptation of arousal levels to meet sensory or behavioral demands. (Aston-Jones and Cohen, 2005; Ferguson and Cardin, 2020; Batista-Brito et al., 2018).

Because pupil size reliably indicates the activation of the LC-NE neurons (Joshi et al., 2016; Varazzani et al., 2015; Murphy et al., 2014), we therefore suggest that increased pupil responses to subthreshold targets reflect a higher demand of NE in order to accommodate arousal levels to satisfy the perceptual and behavioral demands imposed by the thresholded-deviant detection task. This phasic activation of the LC-NE would presumably follos a feedback signal targeting the LC and associated to either higher uncertainty (Urai et al., 2017) or prediction error (Sales et al., 2019) during bottom-up information processing. Interestingly, a plausible neural circuit that could support this feedback mechanism comprises prefrontal and cingulate regions (Aston-Jones and Cohen, 2005). Indeed, direct bidirectional projections exist both between the PFC and the LC (Totah et al., 2020) and the ACC and the LC (Gompf et al, 2010). Temporally, this is also plausible: phasic discharges of NE are reported as fast as 100 milliseconds after LC stimulation and conduction latency to PFC is of 60 milliseconds (Aston-Jones and Cohen, 2005; Aston-Jones et al, 1985), whereas phasic increases in pupil size resulting from LC microstimulation usually start at around 200-250 milliseconds with a mean peak latency between 450-550 milliseconds (Joshi et al., 2016).

In conclusion, we show that the pupil is sensitive to subconscious processing of auditory novelty, reflecting higher activity of the LC-NE system which is necessary for the adaptation of arousal in response to specific task demands. Due to a higher contrast-based saliency, suprathreshold deviant targets were amenable to both automatic orienting-attention mechanisms (i.e. MMN) and executive processes involved in conscious processing (i.e. P3). The desired behavioral output could therefore be obtained without significant changes in the system's arousal levels available upon stimulus presentation. Subthreshold deviant targets, on the other hand, were below thresholds for conscious discrimination and posed a significant perceptual challenge. Although detectable by means 
374 of automatic bottom-up orienting attention mechanisms (i.e. MMN), they escaped higher-order

375 executive processes indexed by the P3 that were required to meet the desired behavioral outcome

376 (deviant detection). This would have resulted in an increase demand of NE to accommodate arousal

377 levels via the adaptation of gain-modulation functions at relevant sensory and attention-mediating

378 cortical area, with the presumed goal of lowering thresholds for conscious identification of

379 subthreshold targets.

381 Methods

382 Participant details: Twenty-four right-handed healthy subjects with no self-reported record of 383 auditory, neurological or neuropsychiatric disorders voluntarily agreed to participate in this study 384 (mean age $=25.5$, range $=13$ ). All participants reported normal hearing and normal or corrected-tonormal vision. Extensive and/or formal musical training, as well as high competence in a second language were considered exclusion criteria. Participants were recruited from among the undergraduate and postgraduate community at Pontificia Universidad Católica de Chile and Universidad de Chile. All participants signed an inform consent prior to their participation in the study.

Procedures and stimuli: Participants sat $50 \mathrm{~cm}$ away from of a screen in a dimly lit room. Participants'

391 brain activity was recorded using a 64-channel Biosemi EEG system and their pupil recorded using 392 an Eyelink 1000 eye-tracking system. The eye-tracking system was calibrated at the beginning of each experimental session. Auditory stimuli were presented binaurally via special airtube earphones (ER-1 Etymotic Research) that minimize electrical interference. Stimuli comprised sequences of 150millisecond long narrowband sinusoidal tones (Table S1) presented with an interstimulus interval of 150 milliseconds. Stimuli were set to be delivered at an intensity of 70dBs. Participants sat within a Faraday cage while performing the task. The task was programmed using the NBS (Neurobehavioral Systems) Presentation software.

Staircase procedure: Because hearing abilities vary across individuals, all participants performed a staircase procedure at the beginning of each experimental block and for each set of stimuli. A 401 sequence of standard tones (dark circles inside dotted box, Figure 1, left) was presented against 402 another sinusoidal tone (the target) $50 \mathrm{~Hz}$ above the standard tone (blue circle). Participant were asked 403 whether the target was the same or different from the preceding tones. If the subject response was 404 "different", a new trial was presented where the target tone was stepped down five $5 \mathrm{~Hz}$ (green arrow, 405 Figure 1, left. But see table S1 for the full set of stimuli). Targets would eventually become 406 increasingly similar to the standard tones (gradient of gray circles). When participants judged a target 
407

408

409

410

411

412

413

414

415

416

417

418

419

420

421

422

423

424

425

426

427

428

429

430

431

432

433

434

435

436

437

438

439

to be the same as the previous standard tones, the subsequent target tone was stepped up in $5 \mathrm{~Hz}$. Once the subject responses entered a same-different response loop (green arrows, Figure 1, middle), the algorithm would identify this region within the staircase as containing the subjects' discriminatory threshold. After four consecutive iterations of this same-different response loop, the subject's discriminatory was set to be the boundary separating the target tone consistently reported as "different" from the target tone consistently reported as "same" (dotted horizontal line, Figure 1, right). Finally, the three stimuli were automatically set: the standard (dark gray circle, tgtSTD), the subthreshold deviant (green circle, subDEV) and the suprathreshold deviant (blue circle, supraDEV). The subthreshold deviant was always set as the tone being two steps bellow the discriminatory threshold and the suprathreshold deviant was always set to be the STD tone plus 50Hz.

Thresholded deviant detection task: The thresholded deviant detection task comprised three blocks. For each block, the base frequency for standard tones would be either $800 \mathrm{~Hz}, 1000 \mathrm{~Hz}$ or $1200 \mathrm{~Hz}$. During each trial, participants listened to sequences of tonal stimuli and were instructed to decide whether the last tone (i.e. the target) was the same or different from the preceding standard tones. Participants had to make their choice by pressing one of two buttons upon appearance of a prompt on screen, 1000 milliseconds after the onset of the target stimulus. This delay in behavioral response was in order to avoid confounding effects due to the temporal and spatial overlap of motor signals and the ERP events of interest. There was no time limit for response. The number of standard tones before each target stimulus randomly varied between three and five tones. Random variability in the number of standard tones before each target was expected to minimize habituation effects. Participants were told to prioritize response accuracy over speed of response. The target stimulus could be either another standard tone (tgtSTD), a tone that was $50 \mathrm{~Hz}$ above the standard tone (supraDEV) or a tone that was below each participants' discriminatory threshold as defined by the staircase procedure (subDEV). The theoretical probability for each type of target was $\sim 33.333 \%$.

Behavioral data analyses: Data $(\mathrm{n}=24)$ was obtained using Presentation software. Default output files were preprocessed and analyzed using in-house Matlab scripts. Each participants' performance was in each block and for each set of stimuli were tested against the chance probability using a binomial test (i.e. the probability of observing $x$ correct or incorrect responses given a theoretical probability $p$ for the corresponding number of trials per block $n$ ). Only data (behavioral, pupil and EEG) from experimental blocks that were above the chance probability were included in further analyzes. We failed to identify the discriminatory threshold of 3 of our participants due to problems during the staircase procedure (e.g. the participant accidentally confused buttons or did not fully understand the task, resulting in unprecise discriminatory threshold that did not reflect their actual 
440

441

442

443

444

445

446

447

448

449

450

451

452

453

454

455

456

457

458

459

460

461

462

463

464

465

466

467

468

469

470

471

472

perceptual abilities, Table S2). Reaction times below zero (i.e. before response prompt appeared on screen) were considered accidental button presses and therefore rejected from analyses. Any reaction time below and above the 0.25 and the 97.5 percentiles at the subject-level were also defined as outliers and therefore rejected. Histograms were plotted to inspect the distribution of reaction times. Because reaction times were right-skewed, approximating a gamma distribution, we computed the median reaction time and used it for subsequent statistical analyses.

EEG data preprocessing: Data $(\mathrm{n}=23)$ was preprocessed using Brainstorm (Tadel et al., 2011, http://neuroimage.usc.edu/brainstorm). EEG data was filtered between 1 and $45 \mathrm{~Hz}$ using a 7426order FIR bandpass filter. Subsequently, data was detrended and visually inspected for noisy channels using Welch's Power Spectrum Density (PSD). Next deleted channels were interpolated and the EEG signal was re-referenced to the average of all electrodes. Oculomotor and blink-related artifacts were removed using and Independent Component Analysis (Makeig et al., 1996) on the continuous EEG signal. Data was epoqued in trials comprising $2500 \mathrm{~ms}$ before and $1000 \mathrm{~ms}$ after presentation of target stimuli. Any trial where the signal exceeded 100 microvolts in amplitude was rejected from subsequent analyses. Event Related Potentials were computed as the baseline-corrected arithmetic average of all individual trials per subject, per target type. Baseline correction was applied by subtracting the mean ERP between -500 milliseconds and time zero. EEG forward models were computed using the symmetric Boundary Method BEM by the open source software OpenMEEG (Gramfort et al. 2010) on default MNI/ICMB152 cortical templates (Fonov et al., 2009) using default Brainstorm parameters. Source estimation was computed using the Minimum Norm solution and unconstrained sLORETA (Pasqual-Marqui, 2002) estimates on the preprocessed data. Matrices for the covariance of all electrodes were computed from approximately 1000ms baseline periods on each epoque. Regions of Interest were selected a priori based on previous literature on auditory mismatch processing as well as on the origins of the MMN/P3 Event-Related potentials, and were manually delimited informed by z-maps shown in figure on the ICBM152 template cortical surface (mean vertices $=188.66$ ). These ROIs were portions of the right and left Superior Temporal Gyrus, right and left Dorsolateral Prefrontal Cortex, bilateral Dorsomedial Prefrontal Cortex and left Anterior Cingulate Cortex. Scalp EEG data from one participant was excluded from analyses due to technical issues during data acquisition.

Pupillometry: Data $(\mathrm{n}=21)$ was acquired using Eyelinks' default acquisition hardware and software at a sampling rate of $1000 \mathrm{~Hz}$. Calibration procedures were carried out at the beginning of each experimental session. Pupil area, horizontal and vertical gaze positions were recorded in a dimly lit room from the right eye of each participant. Blinks and gaze artifacts were detected by Eyelinks' 
473 default algorithms. Pupil data was preprocessed using Urai et al. (2017) pupil pipeline plus additional

474 in-house Matlab script adaptations. Eyelink-defined and additionally detected blinks were padded by

475150 milliseconds and linearly interpolated. The pupil response evoked by blinks and saccadic events

476 was identified via deconvolution and removed using linear regression as in Knapen et al. (2016). The

477 signal was then filtered between $0.01 \mathrm{~Hz}$ and $10 \mathrm{~Hz}$ using a second-order Butterworth filter and then

478 down sampled to $250 \mathrm{~Hz}$. Data was epoqued between 2500 milliseconds before and 1000 milliseconds

479 after the onset of target stimuli and trials where extreme values were below and above the 0.5 and the

48099.5 percentiles were further rejected. Trials were subsequently baseline-normalized ( $\mathrm{z}$ units) and the

481 arithmetic average of the pupil size and its derivative for each target type per participant was

482 estimated. The time window for baseline correction comprised -500 milliseconds to time zero. Pupil

483 data from three participants was unavailable due to technical problems with output data files or trigger

484 coding.

485 Statistics: All statistical analyses were implemented using custom-made Matlab scripts. Abovechance performance was tested using a binomial distribution (binomial test). For tgtSTDs and supraDEVs, the probability of observing $x$ hits given a theoretical probability of 0.5 and $n$ observations, where $n$ is the number of trials per block was tested and data from blocks whose probability was lower than an alpha value of 0.05 were rejected (Table S2). For subDEVs, the probability of observing $x$ incorrect responses given the same theoretical probability and $n$ observations was calculated and the same rejection criterion was applied. For reaction times, we calculated the individual median reaction time per condition. We rejected subjects for which there was no subthreshold deviant data available and performed a two-tailed non-parametric 10.000bootstrap resampling procedure to determine whether there was any statistical difference among conditions. We identified the percentiles corresponding to an alpha level of 0.05 , Bonferronicorrected and compared the against our observed median reaction times. For pupil data, we calculated the arithmetic mean pupil size and mean pupil rate of change across conditions. Data from blocks that failed to meet the above-chance performance criterion were not included. We plotted the times series data per condition with their 95\% confidence intervals for the Standard Error of the Mean. Confidence intervals were calculated using a studentized 5000-bootstrap procedure. We then performed two-

502 the mean values came from the same distribution at an alpha level of 0.05, Bonferroni-corrected.

503 Additionally, we set our algorithm to return only statistical effects that extended for more than 5 504 consecutive timepoints. For scalp ERPs, we performed the same procedure as for pupil data, but 505 instead of performing the resampling procedure during the entire 1000 window, we performed two 506 separate tests for our ERP events of interest (MMN and P3) thusly: a one-tailed 1000-bootstrap 
507

508

509

510

511

512

513

514

515

516

517

518

519

520

521

522

523

524

525

526

527

528

529

530

531

532

533

534

535

536

537

538

539

540

541

542

543

544

545

between 100 and 220 milliseconds and another one-tailed 10000 bootstrap between 200 and 350 milliseconds.

Correlations were conducted using the non-parametric Spearman correlation coefficient test. Subjects for which either pupil data or EEG data was missing were not included in the analyses.

\section{Acknowledgements}

This project was funded by CONICYT-FONDECYT Regular Grant $\mathrm{N}^{\circ} 2831160258$ and ANID/CONICYT National Grant for Doctoral Studies N² 2018-21181786. We would also like to acknowledge Dr. Rodrigo Henriquez-Ch, Dr. Gonzalo Boncompte, Dr. Tomas Ossandón, Vicente Medel, Marcos Domic and Brice Follet for their advice, feedback and support.

\section{References}

1. Ranganath, C., \& Rainer, G. (2003). Neural mechanisms for detecting and remembering novel events. Nature Reviews Neuroscience, 4(3), 193-202.

2. Tiitinen, H., May, P., Reinikainen, K., \& Näätänen, R. (1994). Attentive novelty detection in humans is governed by pre-attentive sensory memory. Nature, 372(6501), 90-92.

3. Sohoglu, E., \& Chait, M. (2016). Detecting and representing predictable structure during auditory scene analysis. Elife, 5, e19113.

4. Töllner, T., Zehetleitner, M., Gramann, K., \& Müller, H. J. (2011). Stimulus saliency modulates pre-attentive processing speed in human visual cortex. PLoS One, 6(1), e16276.

5. Aston-Jones, G., \& Cohen, J. D. (2005). Adaptive gain and the role of the locus coeruleusnorepinephrine system in optimal performance. Journal of Comparative Neurology, 493(1), 99-110.

6. Vazey, E. M., Moorman, D. E., \& Aston-Jones, G. (2018). Phasic locus coeruleus activity regulates cortical encoding of salience information. Proceedings of the National Academy of Sciences, 115(40), E9439-E9448.

7. Ferguson, K. A., \& Cardin, J. A. (2020). Mechanisms underlying gain modulation in the cortex. Nature Reviews Neuroscience, 1-13.

8. Poe, G. R., Foote, S., Eschenko, O., Johansen, J. P., Bouret, S., Aston-Jones, G., ... \& Berridge, C. (2020). Locus coeruleus: a new look at the blue spot. Nature Reviews Neuroscience, 1-16.

9. Quirins, M., Marois, C., Valente, M., Seassau, M., Weiss, N., El Karoui, I., ... \& Naccache, L. (2018). Conscious processing of auditory regularities induces a pupil dilation. Scientific reports, 8(1), 1-11.

10. Zhao, S., Chait, M., Dick, F., Dayan, P., Furukawa, S., \& Liao, H. I. (2019). Pupil-linked phasic arousal evoked by violation but not emergence of regularity within rapid sound sequences. Nature communications, 10. 
11. Bala, A. D., Whitchurch, E. A., \& Takahashi, T. T. (2019). Human Auditory Detection and Discrimination Measured with the Pupil Dilation Response. Journal of the Association for Research in Otolaryngology, 1-17.

12. Liao, H. I., Yoneya, M., Kidani, S., Kashino, M., \& Furukawa, S. (2016A). Human pupillary dilation response to deviant auditory stimuli: Effects of stimulus properties and voluntary attention. Frontiers in Neuroscience, 10, 43.

13. Bekinschtein, T. A., Dehaene, S., Rohaut, B., Tadel, F., Cohen, L., \& Naccache, L. (2009). Neural signature of the conscious processing of auditory regularities. Proceedings of the National Academy of Sciences, 106(5), 1672-1677.

14. Näätänen, R., Paavilainen, P., Rinne, T., \& Alho, K. (2007). The mismatch negativity $(\mathrm{MMN})$ in basic research of central auditory processing: a review. Clinical neurophysiology, 118(12), 2544-2590.

15. Näätänen, R., Kujala, T., \& Light, G. (2019). The Mismatch Negativity (MMN): A window to the brain. Oxford University Press.

16. Garrido, M. I., Kilner, J. M., Stephan, K. E., \& Friston, K. J. (2009). The mismatch negativity: a review of underlying mechanisms. Clinical neurophysiology, 120(3), 453-463.

17. Hyman, J. M., Holroyd, C. B., \& Seamans, J. K. (2017). A novel neural prediction error found in anterior cingulate cortex ensembles. Neuron, 95(2), 447-456.

18. Polich, J. (2007). Updating P300: an integrative theory of P3a and P3b. Clinical neurophysiology, 118(10), 2128-2148.

19. Kamp, S. M., \& Donchin, E. (2015). ERP and pupil responses to deviance in an oddball paradigm. Psychophysiology, 52(4), 460-471

20. Linden, D. E. (2005). The P300: where in the brain is it produced and what does it tell us?. The Neuroscientist, 11(6), 563-576.

21. van der Wel, P., \& van Steenbergen, H. (2018). Pupil dilation as an index of effort in cognitive control tasks: A review. Psychonomic bulletin \& review, 25(6), 2005-2015.

22. Wang, C. A., Boehnke, S. E., Itti, L., \& Munoz, D. P. (2014). Transient pupil response is modulated by contrast-based saliency. Journal of Neuroscience, 34(2), 408-417.

23. Wang, C. A., Baird, T., Huang, J., Coutinho, J. D., Brien, D. C., \& Munoz, D. P. (2018). Arousal effects on pupil size, heart rate, and skin conductance in an emotional face task. Frontiers in Neurology, 9, 1029.

24. Wainstein, G., Rojas-Líbano, D., Crossley, N. A., Carrasco, X., Aboitiz, F., \& Ossandón, T. (2017). Pupil size tracks attentional performance in attention-deficit/hyperactivity disorder. Scientific reports, 7(1), 1-9.

25. Clewett, D., Gasser, C., \& Davachi, L. (2020). Pupil-linked arousal signals track the temporal organization of events in memory. Nature Communications, 11(1), 1-14.

26. Citherlet, D., Boucher, O., Tremblay, J., Robert, M., Gallagher, A., Bouthillier, A., ... \& Nguyen, D. K. (2019). Role of the insula in top-down processing: an intracranial EEG study using a visual oddball detection paradigm. Brain Structure and Function, 224(6), 2045-2059.

27. Han, S. W., Eaton, H. P., \& Marois, R. (2019). Functional fractionation of the Cinguloopercular network: alerting insula and updating cingulate. Cerebral Cortex, 29(6), 26242638.

28. Alamia, A., VanRullen, R., Pasqualotto, E., Mouraux, A., \& Zenon, A. (2019). Pupil-linked arousal responds to unconscious surprisal. Journal of Neuroscience, 39(27), 5369-5376. 
29. Liao, H. I., Kidani, S., Yoneya, M., Kashino, M., \& Furukawa, S. (2016B). Correspondences among pupillary dilation response, subjective salience of sounds, and loudness. Psychonomic bulletin \& review, 23(2), 412-425.

30. Teixeira, M., Castelo-Branco, M., Nascimento, S. \& Almeida, V. (2010). The p300 signal is monotonically modulated by target saliency level irrespective of the visual feature domain. Acta Ophthalmologica, 88.

31. Teixeira, M., Pires, G., Raimundo, M., Nascimento, S., Almeida, V., \& Castelo-Branco, M. (2014). Robust single trial identification of conscious percepts triggered by sensory events of variable saliency. PLOS one, 9(1), e86201.

32. Steiner, G. Z., \& Barry, R. J. (2011). Pupillary responses and event-related potentials as indices of the orienting reflex. Psychophysiology, 48(12), 1648-1655.

33. Harms, L., Parras, G. G., Michie, P. T., \& Malmierca, M. S. (2020). The Role of Glutamate Neurotransmission in Mismatch Negativity (MMN), A Measure of Auditory Synaptic Plasticity and Change-detection. Neuroscience.

34. Murphy, P. R., Robertson, I. H., Balsters, J. H., \& O'connell, R. G. (2011). Pupillometry and $\mathrm{P} 3$ index the locus coeruleus-noradrenergic arousal function in humans. Psychophysiology, 48(11), 1532-1543.

35. Nieuwenhuis, S., Aston-Jones, G., \& Cohen, J. D. (2005). Decision making, the P3, and the locus coeruleus--norepinephrine system. Psychological bulletin, 131(4), 510.

36. Urai, A. E., Braun, A., \& Donner, T. H. (2017). Pupil-linked arousal is driven by decision uncertainty and alters serial choice bias. Nature communications, 8(1), 1-11.

37. de Gee, J. W., Knapen, T., \& Donner, T. H. (2014). Decision-related pupil dilation reflects upcoming choice and individual bias. Proceedings of the National Academy of Sciences, 111(5), E618-E625.

38. Krishnamurthy, K., Nassar, M. R., Sarode, S., \& Gold, J. I. (2017). Arousal-related adjustments of perceptual biases optimize perception in dynamic environments. Nature human behaviour, 1(6), 1-11.

39. Gilzenrat, M. S., Nieuwenhuis, S., Jepma, M., \& Cohen, J. D. (2010). Pupil diameter tracks changes in control state predicted by the adaptive gain theory of locus coeruleus function. Cognitive, Affective, \& Behavioral Neuroscience, 10(2), 252-269.

40. Batista-Brito, R., Zagha, E., Ratliff, J. M., \& Vinck, M. (2018). Modulation of cortical circuits by top-down processing and arousal state in health and disease. Current opinion in neurobiology, 52, 172-181.

41. Joshi, S., Li, Y., Kalwani, R. M., \& Gold, J. I. (2016). Relationships between pupil diameter and neuronal activity in the locus coeruleus, colliculi, and cingulate cortex. Neuron, 89(1), 221-234.

42. Varazzani, C., San-Galli, A., Gilardeau, S., \& Bouret, S. (2015). Noradrenaline and dopamine neurons in the reward/effort trade-off: a direct electrophysiological comparison in behaving monkeys. Journal of Neuroscience, 35(20), 7866-7877.

43. Murphy, P. R., O'connell, R. G., O'sullivan, M., Robertson, I. H., \& Balsters, J. H. (2014). Pupil diameter covaries with BOLD activity in human locus coeruleus. Human brain mapping, 35(8), 4140-4154.

44. Sales, A. C., Friston, K. J., Jones, M. W., Pickering, A. E., \& Moran, R. J. (2019). Locus Coeruleus tracking of prediction errors optimises cognitive flexibility: An Active Inference model. PLoS computational biology, 15(1), e1006267. 
45. Totah, N. K., Logothetis, N. K., \& Eschenko, O. (2020). Synchronous spiking associated with high gamma oscillations in prefrontal cortex exerts top-down control over a $5 \mathrm{~Hz}$ rhythmic modulation of spiking in locus coeruleus. bioRxiv.

46. Gompf, H. S., Mathai, C., Fuller, P. M., Wood, D. A., Pedersen, N. P., Saper, C. B., \& Lu, J. (2010). Locus ceruleus and anterior cingulate cortex sustain wakefulness in a novel environment. Journal of Neuroscience, 30(43), 14543-14551.

47. Aston-Jones G. 1985. Behavioral functions of locus coeruleus derived from cellular attributes. Physiol. Psych. 13:118-26

48. Tadel, F., Baillet, S., Mosher, J. C., Pantazis, D., \& Leahy, R. M. (2011). Brainstorm: a user-friendly application for MEG/EEG analysis. Computational intelligence and neuroscience, 2011.

49. Makeig, S., Bell, A. J., Jung, T. P., \& Sejnowski, T. J. (1996). Independent component analysis of electroencephalographic data. In Advances in neural information processing systems (pp. 145-151).

50. Gramfort, A., Papadopoulo, T., Olivi, E., \& Clerc, M. (2010). OpenMEEG: opensource software for quasistatic bioelectromagnetics. Biomedical engineering online, 9(1), 45.

51. Fonov, V. S., Evans, A. C., McKinstry, R. C., Almli, C. R., \& Collins, D. L. (2009). Unbiased nonlinear average age-appropriate brain templates from birth to adulthood. NeuroImage, (47), S102.

52. Pascual-Marqui, R. D. (2002). Standardized low-resolution brain electromagnetic tomography (sLORETA): technical details. Methods Find Exp Clin Pharmacol, 24(Suppl D), 5-12.

53. Knapen, T., de Gee, J. W., Brascamp, J., Nuiten, S., Hoppenbrouwers, S., \& Theeuwes, J. (2016). Cognitive and ocular factors jointly determine pupil responses under equiluminance. PloS one, 11(5), e0155574. 